Memorias del 1 Congreso Nacional de Investigación en Enseñanza de la Biología. VI Encuentro Nacional de Investigación en Enseñanza de la Biología y la Educación Ambiental. ISSN 2027 1034. P. p. 215- 224.

\title{
UNIDAD DIDÁCTICA PRÁCTICA DOCENTE. \\ ¿CÓMO SOMOS?: UN ACERCAMIENTO A LA
}

\section{DIDACTIC UNIT ¿HOW ARE WE?: AN INTRODUCTION TO TEACHING PRACTICE.}

\section{POR: Juan David Cárcamo}

Seriel Fernando Rincón

Josúe David Rojas ${ }^{1}$

\section{Resumen.}

Se plantea el desarrollo de una Unidad Didáctica (U.D) titulada ¿Cómo somos?, para lo cual, inicialmente se realiza un trabajo de caracterizaciones tanto al plantel educativo, docente titular y estudiantes, además de la realización de un ejercicio de ideas previas, lo cual aportó en la construcción de la Unidad Didáctica. Posteriormente, se abordó el diseño de la U.D teniendo en cuenta la imagen de ciencia y de biología, importancia de biología en la educación, el tema a desarrollar (el cual fue Sistema Endocrino), formas de evaluación y las competencias a desarrollar, comprendiendo la planeación de las sesiones de clases. Finalmente se lleva a cabo la implementación de la U.D en el colegio Madre Paula Montal, con jóvenes de 8 grado de bachillerato con edades que oscilan entre 12 y 14 años. De esta forma se obtuvo como resultado que los estudiantes pasaran de concepciones erróneas hacia un aprendizaje significativo.

Palabras Clave. Aprendizaje significativo, Conceptos, Idea previa, Práctica académica, Sistema endocrino, Unidad didáctica.

\section{Abstract.}

It proposes the development of a Didactic Unit (D.U) entitled ¿Cómo somos? (How are we?), for which initially a characterization work was performed both to the educational institution, teachers and students, in addition to the execution of a previous ideas exercise, which contributed to the construction of the Didactic Unit. Subsequently, the design of the didactic unit was tackled, taking into account the image of science and biology, the importance of biology in education, the topic (the Endocrine System), forms of assessment and competencies to be developed,

1 jdch1988@gmail.com , sefrincon@gmail.com, jeshuandme@gmail.com. Proyecto Curricular Licenciatura en Biología. Universidad Distrital Francisco José De Caldas. Bogotá D.C 2011. 
Memorias del 1 Congreso Nacional de Investigación en Enseñanza de la Biología. VI Encuentro Nacional de Investigación en Enseñanza de la Biología y la Educación Ambiental. ISSN 2027 1034. P. p. 215- 224.

understanding the class sessions planning. Finally, the didactic unit implementation was conducted in the Madre Paula Montal school, with students of 8th grade with ages ranging between 12 and 14 years. Thus it was obtained as a result that students' misconceptions passed from misconceptions to a meaningful learning.

\section{Keywords.}

Meaningful learning, concepts, previous ideas, ,academic practice, endocrine system, didactic unit.

\section{INTRODUCCIÓN}

Guiar el proceso formativo de los estudiantes es la función primordial de un docente, y el cómo lograr una mayor y excelente comprensión, de modo que sean capaces de construir conocimiento, es su mayor cuestionamiento. Es por tal razón que el mejoramiento de la práctica profesional docente va de la mano de la elaboración de una Unidad Didáctica y un análisis profundo de la aplicación de la misma, de modo que se facilite dicho proceso formativo.

De esta forma, además del conocimiento de la disciplina, el profesor debe hacer uso de estrategias que posibiliten y apoyen tanto su enseñanza como el aprendizaje de sus estudiantes y de esta forma el Conocimiento Pedagógico del Contenido (CPC) se constituye como una herramienta ideal para tal fin, ya que en el ejercicio de la docencia es necesaria "la transformación de lo comprendido" de determinado cuerpo disciplinar. O sea, la capacidad de enseñabilidad de determinado contenido descansa, entre otros, en "[...] el conocimiento profundo, flexible y cualificado del contenido disciplinar, pero además, en la capacidad para generar representaciones y reflexiones poderosas sobre ese conocimiento" (Shulman, 1999).

De acuerdo a lo anterior y con el fin de aportar a nuestro proceso formativo como futuros docentes en el área de la Biología, se realiza la implementación de la Unidad Didáctica "¿Cómo Somos?" sobre el tema de Sistema Endocrino. Dicho proceso se llevó a cabo en el Colegio Madre Paula Montal ubicado en el barrio San Rafael, con un grupo de 35 estudiantes del grado $8 \mathrm{~A}$ con edades que oscilan entre los 12-14 años, situados según Piaget (1964) en la última etapa de desarrollo cognitivo: el estadio de las operaciones formales.

Teniendo en cuenta la situación general de los estudiantes en cuanto a su desarrollo cognoscitivo (conocimientos e ideas previas) pero además la disponibilidad de tiempo en el transcurso de nuestra práctica, fue escogido el enfoque de Aprendizaje Significativo propuesto por Ausubel, ya que este presenta la gran ventaja de partir de las ideas previas de los estudiantes para la modificación de la estructura cognoscitiva de éstos por medio de una nueva 
Memorias del 1 Congreso Nacional de Investigación en Enseñanza de la Biología. VI Encuentro Nacional de Investigación en Enseñanza de la Biología y la Educación Ambiental. ISSN 2027 1034. P. P. 215- 224.

información que se integra en sus estructuras de conocimiento, posibilitando así un aprendizaje relevante, integrador y persistente que favorezca la comprensión.

\section{METODOLOGÍA}

La metodología establecida para el desarrollo e implementación de la U.D se dividió en tres fases, caracterización, diseño e implementación, definidas a continuación:

\section{Caracterización}

En esta fase se realizó la descripción del colegio (en cuanto a su PEI, enfoque pedagógico), del docente (enfoque pedagógico, su quehacer y experiencia docente) y de los estudiantes (aspectos socioeconómicos e ideas previas). En el caso de la directora y la docente se efectuó mediante la implementación de una serie de entrevistas y grabaciones de audio y video realizadas en sesiones de clase respectivamente, en el caso de los estudiantes se realizó por medio de un formato tipo encuesta.

\section{Diseño}

En esta fase se estructuró la U.D, para lo cual se hizo necesario la selección del enfoque pedagógico, la definición del eje temático (de acuerdo al plan de estudios de la profesora), las competencias a evaluar, la planeación de las clases (selección de actividades y estrategias de evaluación).

\section{Implementación}

Se desarrolló en 6 clases, siendo estas repartidas en martes ( 1 hora), miércoles (1 hora) y viernes (2 horas), de acuerdo con el horario establecido para la asignatura en este curso.

Clase №1 Acercamiento al sistema endocrino según los estudiantes.

Objetivo: Reconocer de forma grupal cuales son las ideas previas de los estudiantes e iniciar

Clase №2 Las Hormonas y el cuerpo humano.

Objetivo: Permitir por medio de situaciones cotidianas que los estudiantes reconozcan la relación entre las hormonas y el cuerpo humano.

Clase №3 Enfermedades del Sistema Endocrino.

Objetivo: A partir de los casos de la clase anterior y por medio de la aproximación teórica en clase, permitir que los estudiantes reconozcan, argumenten y relacionen, el desarrollo y las clases de enfermedades propias del sistema endocrino.

Clase №4 Hormonas vegetales.

Objetivo: Que los estudiantes reconozcan la importancia de las hormonas en las plantas y de las celúlas que en este caso producen las mismas.

Clase №5 Actividad de repaso general.

Objetivo: Fomentar en los estudiantes la capacidad de analizar y relacionar las actividades, de manera que se complementen entre si. 
Memorias del 1 Congreso Nacional de Investigación en Enseñanza de la Biología. VI Encuentro Nacional de Investigación en Enseñanza de la Biología y la Educación Ambiental. ISSN 2027 1034. P. p. 215- 224.

Clase №6 Evaluación Final

Objetivo: Identificar como los estudiantes por medio de la realización de la actividad van integrar, relacionar y por tal razón a reforzar su estructura con respecto al marco conceptual que han generado.

\section{RESULTADOS}

En lo que corresponde a la caracterización del colegio, de acuerdo a la descripción del PEI, se determinó que éste se basa en Piedad y Letras siguiendo el ejemplo de su patrono San José de calazans. De acuerdo al enfoque pedagógico que maneja el colegio se manisfesto que este se basa en el constructivismo y además siguiendo el ejemplo de Calazans y Paula Montal, que plantean métodos sencillos claros que sean prácticos para el estudiante.

La caracterización de la docente permitió evidenciar las estrategias de su práctica en el aula, basadas en un enfoque de enseñanza expositivo, encaminadas en el desarrollo de la parte del ser, la parte del saber y la parte del hacer.

Finalmente la caracterización de los estudiantes mostró que la edad de ellos oscilaba entre los 12-14 años (Etapa de operaciones formales según Piaget).

En cuanto al diseño de la U.D se consideró pertinente utilizar el enfoque pedagógico de aprendizaje significativo debido a que era el que permitiría obtener mejores resultados teniendo en cuenta la disponibilidad de tiempo para realizar la implementación (el cual era limitado a 6 clases). El diseño de la U.D. obedeció al desarrollo del tema de Sistema Endocrino teniendo en cuenta además aspectos como la imagen de ciencia y biología, la importancia de la biología en la educación, el objetivo de nuestra práctica docente, el enfoque, la competencia y el desarrollo conceptual del tema por medio de las actividades propuestas en cada sesión de clase.

Con respecto a la implementación, la primera sesión se abordó desde las concepciones de los estudiantes, en la cual encontramos que sus ideas previas estaban limitadas a relacionar el funcionamiento del sistema endocrino a cierta etapa de la vida (la adolescencia), además que no se distinguieron los órganos que intervenían en dicho sistema.

Alternativamente, para socializar las ideas previas entre los estudiantes del grupo se realizó un mapa conceptual grupal a partir de los conceptos que los estudiantes planteaban respecto a sistema endocrino, sus órganos y sus funciones, lo que nos permitió corroborar que efectivamente la mayoría de los estudiantes desconocían los aspectos generales de este.

En cuanto a las sesiones siguientes se realizó un acercamiento a los conceptos de glándulas, hormonas, enfermedades del sistema endocrino y hormonas vegetales, de forma que los cuestionamientos surgidos en cada uno de los temas condujeran hacia la respuesta esperada por medio de la guía del docente, lo cual es posible evidenciar a continuación en las respuestas dadas a las preguntas : ¿Por qué la 
Memorias del 1 Congreso Nacional de Investigación en Enseñanza de la Biología. VI Encuentro Nacional de Investigación en Enseñanza de la Biología y la Educación Ambiental. ISSN 2027 1034. P. p. 215- 224.

ubicación de las glándulas? inicialmente no hay respuesta, pero preguntando, ¿Por qué el cerebro tiene esa ubicación? la respuesta es "porque controla el cuerpo", de esa manera se llega a la respuesta, que "las glándulas tienen una ubicación específica para cumplir determinada función"; ¿Qué son las hormonas? Donde respondieron, "son mensajeros químicos", a continuación se les preguntó ¿Qué quiere decir que son mensajeros? a lo cual contestaron, "que se transportan de un lugar a otro", la pregunta a continuación fue ¿Por qué se transportan? a lo cual respondieron "para llevar una información de un lugar a otro, para cumplir determinada función".

Posteriormente se desarrolló una actividad expositiva por parte de los estudiantes donde se quería evidenciar la competencia explicativa, la cual según el ICFES se refiere a "la capacidad para construir y comprender argumentos, representaciones o modelos que den razón de fenómenos", partiendo de las explicaciones dadas en las sesiones anteriores de clases. La actividad consistió en la elaboración de un esquema del cuerpo humano donde se representó el sistema endocrino, en el cual debían dar cuenta de los conceptos vistos en clase en cuanto a glándulas, hormonas y su función e incidencia sobre el cuerpo humano.

Finalmente en la evaluación se intentó realizar una valoración de los procesos de enseñanza-aprendizaje por medio de preguntas relacionadas al sistema endocrino, otra pregunta referente a su punto de vista sobre la importancia del conocimiento de este y finalmente la elaboración de un mapa conceptual en el que establecieran relaciones sobre los conceptos del tema, evidenciando de esta forma si los estudiantes han entendido realmente los significados básicos que se les ha intentado enseñar, logrando un aprendizaje significativo, y además de comprobar el grado de argumentación por medio de la competencia "explicativa" que nos propusimos desarrollar.

\section{DISCUSIÓN}

Debido a que el interés de este trabajo es mostrar el aporte de la U.D a la práctica docente, como herramienta que posibilita la investigación-acción en el aula, la discusión está orientada a dar cuenta de la etapa de Implementación de la U.D. Esta fase permitió un primer acercamiento a la labor docente, la planeación de clases y la experiencia en el aula, importante en nuestra formación como futuros docentes.

En un primer momento, se hizo necesaria la valoración de las ideas previas de los estudiantes, ya que como plantea Ausubel (1981) "Si tuviese que reducir toda la psicología educativa a un solo principio, enunciaría este: el factor más importante que influye en el aprendizaje es lo que el alumno ya sabe. Averígüese esto y enséñese consecuentemente". Teniendo en cuenta que en la primera actividad de reconocimiento de ideas previas la profesora intervino, es posible decir que se vio intervenida negativamente, ya que la profesora no actúo como una mediadora en 
Memorias del 1 Congreso Nacional de Investigación en Enseñanza de la Biología. VI Encuentro Nacional de Investigación en Enseñanza de la Biología y la Educación Ambiental. ISSN 2027 1034. P. p. 215- 224.

la identificación de los conocimientos previos (Ahumada, 2001) y en consecuencia optó por sugerir responder "No sé", lo cual se vio reflejado en la mayoría de las respuestas de los estudiantes.

Pese al primer inconveniente y de acuerdo con la actividad de socialización de ideas previas es posible afirmar que sí hay un estado inicial del cual partir, de manera tal que es posible establecer relaciones con los nuevos conocimientos para así evidenciar un nivel de significancia, ya que las ideas previas son entendidas generalmente como conceptos científicos erróneos (Fernández, 2002), y poseen una gran relevancia ya que "lo más importante entre lo que se enseña y lo que se conoce es lo que ya se sabe" (Fernández, 2002), debido a que es sólo cuando la nueva información se asimila y se une a un estructura cognitiva preexistente que se produce el aprendizaje (Penchansky, 2004).

La socialización de las ideas previas de los estudiantes resultó ser un método por el cual los estudiantes vinculaban los nuevos conceptos aplicados por ellos mismos a sus estructuras preconcebidas, trayendo como ejemplo las etapas de la vida planteando como cambio que no solo en la adolescencia se presentan cambios sino que estos cambios se ven regulados durante toda la vida, y que estos cambios son debido a mensajes químicos llamados hormonas, que son producidas por una serie de glándulas que están presentes en todo el cuerpo y que en conjunto interaccionan con los demás sistemas creando así una relación generalizada con las demás partes del cuerpo, rompiendo por ende el concepto de que cada sistema es individual y acoplando la idea integradora de que todos tienen una relación entre sí.

La dinámica de las clases permitió a los estudiantes avanzar hacia un aprendizaje significativo, partiendo de uno por descubrimiento por lo menos en una fase inicial, en cuanto a que las respuestas a las preguntas realizadas durante la clase, fueron encontradas por ellos guiados por el docente, como plantea Ausubel (1981) "el rasgo esencial del aprendizaje por descubrimiento es que el contenido principal de lo que va a ser aprendido no se da, sino que debe ser descubierto por el alumno antes de que pueda incorporar lo significativo de la tarea a su estructura cognoscitiva", según la "evolución" de las respuestas se hace evidente que hubo por parte de los estudiantes ésta fase, pero como plantea el mismo Ausubel "Después de realizado el aprendizaje por descubrimiento el contenido descubierto se hace significativo, en gran parte, de la misma manera que el contenido presentado se hace significativo en el aprendizaje por recepción (Por repetición o significativo) (Ausubel, 1981). 
Memorias del 1 Congreso Nacional de Investigación en Enseñanza de la Biología. VI Encuentro Nacional de Investigación en Enseñanza de la Biología y la Educación Ambiental. ISSN 2027 1034. P. p. 215- 224.

Fue importante dar a conocer a los estudiantes que a pesar de que las plantas no poseen glándulas especializadas, si existen células capaces de producir hormonas que regulan procesos de la planta, ya que de esta forma se pudo establecer una relación entre los procesos llevados a cabo en el hombre y en las plantas, esto por medio de la enseñanza expositiva, por que como nos plantea Pozo la enseñanza expositiva "es un modelo eficaz para lograr un ajuste progresivo de las concepciones de los alumnos al conocimiento científico" (Pozo, Gómez, 2006), y para ello se utilizó el concepto de hormona utilizado anteriormente, de modo que los estudiantes pudieran definir qué: " las plantas regulan sus funciones fisiológicas por medio de hormonas que son transportadas por el xilema y el floema" y que además estas "dependen de procesos externos". Teniendo en cuenta los conocimientos previos del estudiante es entonces factible asegurar una conexión entre los nuevos conocimientos y él (Pozo, Gómez, 2006).

En la actividad planteada para desarrollar la competencia explicativa se propuso evidenciar la construcción conceptual y explicativa por medio de una enfermedad endocrina que diera cuenta del funcionamiento hormonal, su acción y efectos sobre el organismo como regulador de distintas funciones. En esta actividad se vio que los estudiantes eligieron enfermedades que algún familiar padecía o en determinados casos ellos mismos, lo cual permite un acercamiento más directo al conocimiento científico desde situaciones cotidianas previas, con lo cual es más factible generar nuevas explicaciones que se acoplen con mayor facilidad a las concepciones previas que tengan sobre estas enfermedades, de forma similar a como las ideas previas sirven de soporte para la reestructuración de nuevos conocimientos.

En la evaluación hemos utilizado el caso de 3 estudiantes: En el primer caso (A) (ver anexo 1.) el estudiante identifica al sistema endocrino como aquel que "regula funciones" sin embargo es una explicación muy general que no ahonda en los aspectos específicos y particulares del tema. Tampoco se evidencia una diferenciación progresiva de los conceptos, de forma que, como propone Novak, "los nuevos conceptos alcancen mayor significado a medida que se adquieren nuevas relaciones, por tanto, los conceptos nunca se aprenden totalmente, sino que se están aprendiendo, modificando o haciendo más explícitos a medida que se van diferenciando progresivamente" (Ontoria, 2006). De esta forma, las jerarquizaciones dadas a conceptos de "hormonas" y "plantas" están en el mismo nivel, luego de "sistema endocrino". En el mapa conceptual, no hay relaciones cruzadas entre conceptos, de forma que por ejemplo, el concepto de "glándulas" no se asocia con el término "hormonas" en ningún momento. 
Memorias del 1 Congreso Nacional de Investigación en Enseñanza de la Biología. VI Encuentro Nacional de Investigación en Enseñanza de la Biología y la Educación Ambiental. ISSN 2027 1034. P. p. 215- 224.

Para el caso B (ver anexo 2.) encontramos que se identifica la participación del sistema endocrino en funciones del cuerpo nombradas por el estudiante como "sexual", "desarrollo", "forma de ser", "crecer" y además relaciona éstas con el concepto hormona, por lo cual reconoce el papel hormonal en la regulación del funcionamiento del cuerpo en varios aspectos. El estudiante también señala que las glándulas producen hormonas, aunque invierte la jerarquía conceptual en la medida que parte de un concepto específico del sistema endocrino (hormona) hacia uno más general (glándula, puesto que no todas las glándulas pertenecen a dicho sistema). También, no hace la salvedad de que las glándulas exocrinas no son parte del sistema endocrino. Otra observación importante es el hecho de que fracciona la parte relacionada con las fitohormonas con el mapa conceptual del sistema endocrino, quedando ambos incomunicados y sin relación alguna. Sin embargo, hace uso mínimo de palabras-enlace para indicar las relaciones conceptuales.

En el caso C (ver anexo 3), vemos que el estudiante ha alcanzado un mayor grado explicativo y de relación conceptual a pesar que inicialmente no evidenció conocimiento previo alguno sobre el tema. En su construcción conceptual se evidencia una correcta conexión entre conceptos y además una jerarquización que parte de lo general a lo específico, sin embargo el estudiante no utiliza palabras enlace en las relaciones conceptuales ni existen relaciones cruzadas que muestren interacciones entre diferentes partes del mapa conceptual.

Respecto a estos tres casos se puede agregar que para que exista un aprendizaje significativo, "el alumno debe manifestar una actitud hacia el aprendizaje significativo; es decir una disposición para relacionar, no arbitraria, sino sustancialmente, el material nuevo con su estructura cognoscitiva, como que el material que aprende es potencialmente significativo para él, especialmente relacionable con su estructura de conocimiento, de modo intencional y no al pie de la letra, (Ausubel citado por Zubiría, 1961), de acuerdo a lo anterior tanto en el caso A y B los estudiantes, posiblemente no poseían la disposición de un aprendizaje significativo, lo que pudo conllevar, que su aprendizaje fuese arbitrario de modo que no hubiese generado ningún tipo de relación que permitiera conllevar a un aprendizaje significativo, cosa que se ve reflejada en la pobreza conceptual mayormente marcada en el caso $A$, pero que es posible evidenciar de igual manera en el caso B.

Por el contrario en el caso $\mathrm{C}$ se observa una gran evolución conceptual, que permite reconocer que si existe en el estudiante la disposición de aprender de forma significativa, se puede lograr un aprendizaje de este modo, ya que se puede pasar de un significado lógico a uno psicológico en el estudiante. 
Memorias del 1 Congreso Nacional de Investigación en Enseñanza de la Biología. VI Encuentro Nacional de Investigación en Enseñanza de la Biología y la Educación Ambiental. ISSN 2027 1034. P. P. 215- 224.

\section{CONCLUSIONES}

En el enfoque de Aprendizaje significativo trabajado, se propone una enseñanza de las ciencias que partiera de la "transformación del significado lógico en significado psicológico", la cual se ve manifestada en la necesidad de que los estudiantes puedan acercar sus ideas previas siendo estas contrastadas con un significado científico, con el fin de generar una apropiación de tales significados, por medio de un proceso de estructuración del pensamiento.

Podemos decir que se establecieron algunos marcos referenciales con los cuales los estudiantes se sintieron identificados en cuanto a que hablamos de temas tales como: los cambios físicos y psicológicos debido al aumento de las secreciones hormonales, algunas de las enfermedades que eran cercanas a su vida cotidiana, los factores que hacen que una planta crezca, aspectos que facilitaron la significancia de estos contenidos en la medida que eran más relacionables con sus propias experiencias.

De esta forma, la construcción conceptual del estudiante fue evidenciada por medio de los mapas conceptuales, de acuerdo al número de relaciones que establecieron entre los conceptos trabajados en los diferentes temas propuestos y además en la relevancia de los contenidos aprendidos.

Como dificultades en la implementación de la U.D podemos señalar la desmotivación de algunos de los estudiantes en la realización de las actividades, lo cual puede afectar la comprensión en la medida que no exista una disposición para el aprendizaje. También, señalamos lo propuesto por Pozo en cuanto que en el enfoque de Aprendizaje Significativo si bien permite un ajuste progresivo de las concepciones de los alumnos al conocimiento científico, plantea que no es suficiente para lograr la reestructuración de estas concepciones, donde los procesos de integración conceptual resultan más difíciles que los de diferenciación.

Es así que pese a las dificultades, fue posible apreciar por parte de los estudiantes la asimilación de los nuevos conocimientos propuestos y su contrastación con los saberes previos, su acercamiento con situaciones cercanas a ellos y el entendimiento de los conceptos involucrados en el desarrollo del tema de sistema endocrino, su relación conjunta e interdependiente 
Memorias del 1 Congreso Nacional de Investigación en Enseñanza de la Biología. VI Encuentro Nacional de Investigación en Enseñanza de la Biología y la Educación Ambiental. ISSN 2027 1034. P. p. 215- 224.

\section{BIBLIOGRAFÍA}

- Ahumada, P (2001). La evaluación en una concepción en aprendizaje significativo. Ediciones Universitarias de Valparaíso. Chile.

- Ausubel, D. (1981). Psicología Educativa. (2a Edición). México: Editorial Trillas.

- Fernández, M. (2002). Algunas consideraciones para la utilización de las ideas previas en la enseñanza de las ciencias morfológicas veterinarias. Revista Electrónica de Enseñanza de las Ciencias, Vol. 1, № 3, 141-152. Universidad de Granma, Cuba.

- Giordan, A. (1993). La enseñanza de las ciencias siglo XXI. España: España Editores.

- - Penchansky, L (2004). El nivel inicial. Estructuración. Orientación para la práctica. Ediciones Colihue. Buenos Aires, Argentina

- Piaget, J. (1964). Seis estudios de psicología.

- Ontoria, A. (2006). Mapas conceptuales: una técnica para aprender. España: Narcea.

- Pozo, J.I.; Gómez, M.A. (2006). Aprender y enseñar ciencia: del conocimiento cotidiano al conocimiento científico" 277-286, 297. España: Morata.

- Shulman, L. (1999). Forward. In J.Gess-Newsome and N.G. Lederman (Eds), Examining Pedagogical Content Knowledge: The Construct and its Implications for Science Teaching (pp. ix-ii). Dordrecht: Kluwer.

- Zubiría, J. (2006). Los modelos pedagógicos. Hacia una pedagogía dialogante. 164-165. Cooperativa Editorial Magisterio: Colombia. 